\title{
EVALUATION OF THE CONCORDANCE DIAGNOSTIC IN RHEUMATIC DISEASES BETWEEN THE PRIMARY AND TERTIARY LEVELS OF HEALTH CARE
}

Maria Eugênia Farias Teixeira (Hospital das Clínicas de Ribeirão Preto, Ribeirão Preto, SP, Brasil), Saulo Musse Dib (Hospital das Clínicas de Ribeirão Preto, Ribeirão Preto, SP, Brasil), Letícia Pastorelli Bonjorno (Hospital das Clínicas de Ribeirão Preto, Ribeirão Preto, SP, Brasil), Samara Libich Gusmão Gigante (Hospital das Clínicas de Ribeirão Preto, Ribeirão Preto, SP, Brasil), Pâmella Indira da Silva Oliveira Menezes (Hospital das Clinicas de Ribeirão Preto, Ribeirão Preto, SP, Brasil), Renê Donizeti Ribeiro de Oliveira (Hospital das Clínicas de Ribeirão Preto, Ribeirão Preto, SP, Brasil), Paulo Louzada Junior (Hospital das Clínicas de Ribeirão Preto, Ribeirão Preto, SP, Brasil)

\section{BACKGROUND}

Musculoskeletal symptoms are the leading cause of demand for medical care at the primary health care level, where physicians often have insufficient skills diagnose and treat rheumatic diseases. The aims were to evaluate the diagnostic concordance of rheumatic diseases between the primary and tertiary health care levels by comparing the diagnosis present in the Public Health System (PHS) referral letters and the diagnosis obtained by the rheumatologists from a tertiary service.

\section{MATERIALS AND METHODS}

Diagnoses of referral letters were compared with those obtained by two groups of rheumatologists at the tertiary service: trainee and senior. Patients were enrolled from August 2018 to December 2018, with clinical follow-up data until 25/03/2019. Sensitivity (S), specificity (E), positive predictive value (PPV) and Kappa ( $k$ ) coefficient of the two comparisons were obtained, with the senior evaluation as the gold standard.

\section{RESULTS}

231 were summoned, if which 31 did not attend. Of the 200 evaluated, 17 lost follow-up, 52 are still under investigation and 131 received a definitive diagnosis. The prevalence of rheumatic diseases was: rheumatoid arthritis (RA), 17.6\%; fibromyalgia, 16.9\%; osteoarthritis, $16.19 \%$; spondyloarthritis (Spa), 8.45\%; systemic lupus erythematosus. $8.45 \%$; crystal arthritis, $4.9 \%$; systemic sclerosis, $4.22 \%$; Sjögren's syndrome, $3.52 \%$; rheumatism of soft parts, $3.5 \%$; vasculitis, $2.1 \%$ and $7 \%$ for absence of rheumatic disease. Grouping the diseases by pathophysiology and similarities in systemic involvement, we find the following values of $S, E, P P V$ and $k$, respectively, for basic care diagnoses: non-inflammatory rheumatic diseases $(0.17,0.94,0.73 ; 0,12)$, RA and Spa $(0.65,0.67,0.43,0.27)$; systemic connective tissue diseases and vasculitis $(0.59,0.90,0.61,0.5)$ and non-rheumatic diseases $(0.2,0.97,0.4,0.23)$. When comparing the diagnoses made by rheumatology physicians and senior rheumatologists, we found: noninflammatory rheumatic diseases $(0.79,0.96,0.94,0.75)$; RA and Spa $(0.89,0.95,0.87,0.83)$; systemic connective tissue diseases and vasculitis $(0.96,0.98,0.93,0.93)$ and non-rheumatic diseases $(0.8,1.1$; 0.88). Confronting the diagnoses obtained by trainee with those of the referral letter, the results of $S, E$, PPV and $k$ were similar to those of the comparison of those with the seniors.

\section{CONCLUSION}

The tertiary rheumatology department receives many cases of low complexity, which should be kept in primary care. The degree of diagnostic agreement between the primary care physicians and the rheumatology team was low, demonstrating the need to improve the medical teaching of rheumatic diseases. Doctors in training in the tertiary service present high agreement of diagnoses when confronted with senior doctors. 